\title{
AUTHOR INDEX VOLUME 11 (2002)
}

Abdeldayem H, see Kukhtarev $N$

Assanto G, see Conti C

Banerjee PP, see Kukhtarev $N$

Boyd RW, see Lukishova SG

Caputo R, Sukhov AV, Tabiryan NV \& Umeton C, Distinct Dynamic Regimes in a Material System with Optically Modulated Diffusivity

Carbone F, see Tartara $L$

Chen Y, see Nagamura T

Clays K, Wostyn K, Zhao Y \& Persoons A, Alternative Experimental Determination of Weak Localization of Light in Nanostructured Materials

$11(2002) 25$

Conti C, Assanto G \& Trillo S, Gap Solitons and Slow Light

Cristiani I, see Tartara $L$

Crookston MB, see Russell TH

Cross GH, see Ren $Y$

Degiorgio V, see Tartara $L$

Deykoon AM, Soskin MS \& Swartzlander, Jr. GA, Nonlinear Cusp Diffraction Catastrophe and Vortex Quadrupoles from a Smooth Initial Beam

$11(2002) 351$

Di Fabrizio M, see Lucchetti $L$

Ding YJ \& Zotova IB, Coherent and Tunable Terahertz Oscillators, Generators, and Amplifiers

Dupont E, see Liu HC

Edwards ME, see Kukhtarev $N$

Ershov M, see Liu HC

Faccio D, see Tartara $L$

Francescangeli O, see Lucchetti $L$

Frazier D, see Kukhtarev $N$

Friday WA, see Kukhtarev $N$

Fuh AY-G, Liao C-C, Hsu K-C, Lu C-L \& Mo T-S, Laser-Induced Ripple Structure of Dopant on the Substrates in a Dye-Doped Liquid Crystal Cell and Its Alignment Effect

$11(2002) 57$

Ganapathy R \& Kuriakose VC, Soliton Pulse Compression in a Dispersion Decreasing Elliptic Birefringent Fiber with Effective Gain and Effective Phase Modulation

11 (2002) 261

$11(2002) 239$

11 (2002) 75

Hanack M, see Nagamura T

Hanamura E \& Tanabe Y, Nonlinear Optical Responses of Antiferromagnetic Insulators

Hees U, see Zong $Y$

Heinz TF, see Shan $J$

Hsu K-C, see Fuh $A Y-G$

Huang P-R, Lin T-S \& Lue J-T, Nonlinear Dynamic Oscillations of Optical Transmittance for Nematic Liquid Crystals After the Turn-Off of Applied Electric Fields

$11(2002) 153$

Hudson T, see Kukhtarev $N$

Jefimovs K, see Tuovinen $H$

11 (2002) 185

11 (2002) 99 
Kaino T, see Taima T

Kauranen M, see Tuovinen $H$

Kawata S, see Sukhorukov AA

Keller O, Nonlinear Optics in the Near-Field Zone of Atoms

$11(2002) 275$

Khoo IC, see Shishido A

Kivshar YS, see Sukhorukov AA

Knoll W, see Zong $Y$

Komatsu K, see Taima T

Kukhtarev N, Kukhtareva T, Edwards ME, Penn B, Frazier D, Abdeldayem H, Banerjee PP, Hudson T \& Friday WA, Photoinduced Optical and Electrical High-Voltage Pulsations and Pattern Formation in Photorefractive Crystals

$11(2002) 445$

Kukhtareva T, see Kukhtarev $N$

Kuriakose VC, see Ganapathy $R$

Lemmetyinen $\mathrm{H}$, see Tuovinen $H$

Lepeshkin N, see Lukishova $S G$

Li C-F, Zhou F \& Yang K, High Accuracy Optical Bistable Interferometric Fiber Sensors

Liao C-C, see Fuh $A Y$-G

Lin T-S, see Huang $P-R$

Liu HC, Dupont E \& Ershov M, Nonlinear Quantum Well Infrared Photodetector

$11(2002) 433$

Liu X, see Wise F

Lu C-L, see Fuh $A Y$ - $G$

Lucchetti L, Di Fabrizio M, Francescangeli O \& Simoni F, Light-Induced Adsorption and Desorption in Dynamic and Stable Grating Formation in Methyl-Red Doped Liquid Crystals

Lue J-T, see Huang $P$ - $R$

Lukishova SG, Boyd RW, Lepeshkin N \& Marshall KL, Cumulative Birefringence Effects of Nanosecond Laser Pulses in Dye-Doped Planar Nematic Liquid Crystal Layers

$11(2002) 341$

Marshall KL, see Lukishova $S G$

Mo T-S, see Fuh $A Y$ - $G$

Nagamura T, Naito A, Yoshida I, Chen Y \& Hanack M, All-Optical Reflectance Control Based on Photoinduced Complex Refractive Index Changes in Guided Mode Thin Films Containing Indium or Gallium Phthalocyanines

$11(2002) 205$

Nahata A, see Shan J

Naito A, see Nagamura T

Nakkeeran K, Dark Solitons in Optical Fibers with Higher Order Effects

Ono H, Detailed Characteristics of Photothermal Index Change in Guest-Host Liquid Crystals

Palacios SL, An Explicit Calculation of the Dispersive and Nonlinear Frequency Chirps for Femtosecond Optical Pulses of the High Dispersive Cubic and Cubic-Quintic Nonlinear Schrödinger Equations

$11(2002) 13$

Penn B, see Kukhtarev N

Persoons A, see Clays $K$

Podolskiy VA, Sarychev AK \& Shalaev VM, Erratum to: Plasmons Modes in Metal Nanowires and Left-Handed Materials

$11(2002) 197$

$11(2002) 219$

$11(2002) 143$

$11(2002) 339$ 
Podolskiy VA, Sarychev AK \& Shalaev VM, Plasmon Modes in Metal Nanowires and Left-Handed Materials

$11(2002) 65$

Qian L, see Wise F

Rühe J, see Zong $Y$

Ren Y \& Cross GH, Photostability Studies on High Dipole Nonlinear Optical Adducts of TCNQ in Polymer Optical Waveguides

$11(2002) 131$

Roh WB, see Russell TH

Romagnoli M, see Tartara $L$

Russell TH, Willis SM, Crookston MB \& Roh WB, Stimulated Raman Scattering in Multi-Mode Fibers and Its Application to Beam Cleanup and Combining

Sarychev AK, see Podolskiy VA

Sarychev AK, see Podolskiy VA

Shalaev VM, see Podolskiy VA

Shalaev VM, see Podolskiy VA

Shan J, Nahata A \& Heinz TF, Terahertz Time-Domain Spectroscopy Based on Nonlinear Optics

Sharma SK, Sharma VK \& Tripathi KN, Fabrication and

Characterization of Multilayer Waveguide for Integrated Optics

Sharma VK, see Sharma SK

Shih M-Y, see Shishido A

Shishido A, Shih M-Y \& Khoo IC, Analysis of Optically Induced

Refractive Index Change of Dye-Doped Nematic Liquid Crystals

Shoji S, see Sukhorukov AA

Simoni F, see Lucchetti $L$

Soskin MS, see Deykoon AM

Sukhorukov AA, Shoji S, Kivshar YS \& Kawata S, Self-Written

Waveguides in Photosensitive Materials

Sukhov AV, see Caputo $R$

Swartzlander, Jr. GA, see Deykoon $A M$

Tabiryan NV, see Caputo $R$

Taima T, Komatsu K \& Kaino T, Novel Crystallization Method: Ring-Heater Heated Pedestal Growth Method for Nonlinear Optical Organic Material

$11(2002) 303$

$11(2002) 31$

$11(2002) 173$

$11(2002) 1$

$11(2002) 391$

Tanabe Y, see Hanamura E

Tartara L, Cristiani I, Degiorgio V, Carbone F, Faccio D \& Romagnoli M, Nonlinear Propagation of Ultrashort Laser Pulses in a Microstructured Fiber

$11(2002) 409$

Tkachenko NV, see Tuovinen $H$

Trillo S, see Conti $C$

Tripathi KN, see Sharma SK

Tuovinen H, Kauranen M, Jefimovs K, Vahimaa P, Vallius T, Turunen J, Tkachenko NV \& Lemmetyinen H, Linear and Second-Order Nonlinear Optical Properties of Arrays of Noncentrosymmetric Gold Nanoparticles

Turunen J, see Tuovinen $H$

Umeton C, see Caputo $R$

Vahimaa $\mathrm{P}$, see Tuovinen $H$

Vallius T, see Tuovinen $H$

Wang $\mathrm{B}$, see Wang $R$ 
Wang R, Wei Y-D \& Wang B, Photorefractive Effect of Ce:Fe:LiNbO 3 Crystal

$11(2002) 179$

Wei Y-D, see Wang $R$

Willis SM, see Russell TH

Wise F, Qian L \& Liu X, Applications of Cascaded Quadratic

Nonlinearities to Femtosecond Pulse Generation

11 (2002) 317

Wostyn K, see Clays K

Yang K, see Li C-F

Yoshida I, see Nagamura T

Zhao Y, see Clays $K$

Zhou F, see Li $C$-F

Zong Y, Hees U, Knoll W \& Rühe J, Photoreactive Thin Films of Azobenzene-Derivatized Poly(amic acid) and Poly(imide)

Langmuir-Blodgett-Kuhn Multilayer Assemblies

11 (2002) 367

Zotova IB, see Ding $Y J$ 